\title{
BMJ Global Health Does community-based distribution of HIV self-tests increase uptake of HIV testing? Results of pair-matched cluster randomised trial in Zambia
}

\author{
Melissa Neuman (D) , ${ }^{1}$ Bernadette Hensen, ${ }^{2}$ Alwyn Mwinga ${ }^{3}$ Namwinga Chintu,,${ }^{4,5}$ \\ Katherine L Fielding, ${ }^{1,6}$ Nixon Handima, ${ }^{3}$ Karin Hatzold, ${ }^{5}$ Cheryl Johnson, ${ }^{7}$ \\ Chama Mulubwa, ${ }^{3}$ Mutinta Nalubamba, ${ }^{4}$ Eveline Otte im Kampe, ${ }^{1}$ \\ Musonda Simwinga, ${ }^{3}$ Gina Smith, ${ }^{4,5}$ Dickson Tsamwa, ${ }^{3}$ Elizabeth Lucy Corbett, ${ }^{2,8}$ \\ Helen Ayles ${ }^{2,3}$
}

To cite: Neuman $M$, Hensen B, Mwinga A, et al. Does community-based distribution of HIV self-tests increase uptake of HIV testing? Results of pair-matched cluster randomised trial in Zambia. BMJ Global Health 2021;6:e004543. doi:10.1136/ bmjgh-2020-004543

Handling editor Seye Abimbola

- Additional material is published online only. To view, please visit the journal online (http://dx.doi.org/10.1136/ bmjgh-2020-004543).

Received 24 November 2020 Revised 20 January 2021 Accepted 30 January 2021

Check for updates

(c) World Health Organization 2023. Licensee BMJ.

For numbered affiliations see end of article.

Correspondence to Dr Melissa Neuman; Melissa.Neuman@Ishtm.ac.uk

\section{ABSTRACT}

Objectives Ending HIV by 2030 is a global priority. Achieving this requires alternative HIV testing strategies, such as HIV self-testing (HIVST) to reach all individuals with HIV testing services (HTS). We present the results of a trial evaluating the impact of community-based distribution of HIVST in community and facility settings on the uptake of HTS in rural and urban Zambia.

Design Pair-matched cluster randomised trial.

Methods In catchment areas of government health facilities, OraQuick HIVST kits were distributed by community-based distributors (CBDs) over 12 months in 2016-2017. Within matched pairs, clusters were randomised to receive the HIVST intervention or standard of care (SOC). Individuals aged $\geq 16$ years were eligible for HIVST. Within communities, CBDs offered HIVST in high traffic areas, door to door and at healthcare facilities. The primary outcome was self-reported recent testing within the previous 12 months measured using a population-based survey.

Results In six intervention clusters (population 148541 ), 60 CBDs distributed 65585 HIVST kits. A recent test was reported by $66 \%(1622 / 2465)$ in the intervention arm compared with 60\% (1456/2429) in SOC arm (adjusted risk ratio $1.08,95 \% \mathrm{Cl} 0.94$ to $1.24 ; \mathrm{p}=0.15)$. Uptake of the HIVST intervention was low: $24 \%$ of respondents in the intervention arm (585/2493) used an HIVST kit in the previous 12 months No social harms were identified during implementation. Conclusion Despite distributing a large number of HIVST kits, we found no evidence that this community-based HIVST distribution intervention increased HTS uptake. Other models of HIVST distribution, including secondary distribution and community-designed distribution models, provide alternative strategies to reach target populations.

Trial registration number ClinicalTrials.gov Registry (NCT02793804)

\section{INTRODUCTION}

HIV testing services (HTS) are the gateway to HIV prevention and treatment services. Without increased coverage of HTS, global

\begin{abstract}
WHAT IS ALREADY KNOWN?
$\Rightarrow$ HIV testing coverage remains low in many populations in Southern Africa, and new strategies are needed to increase testing uptake particularly in settings and among populations where the number of people with undiagnosed HIV and new infections is high.

$\Rightarrow$ HIV self-testing (HIVST) is acceptable in many populations and can be a tool for increasing access to testing.

\section{WHAT ARE THE NEW FINDINGS?}

$\Rightarrow$ After 12 months of a pragmatic HIVST distribution programme in communities in rural and urban Zambia, we found no evidence of an increase in population testing coverage in the general adult population, among men, or among young people.

$\Rightarrow$ In intervention areas, many people had not heard of HIVST or seen an HIVST community-based distributor in their community, suggesting that the intervention may not have reached all population members.

WHAT DO THE NEW FINDINGS IMPLY?

$\Rightarrow$ When new HIV testing strategies are implemented, rigorous monitoring and evaluation of activities in real time, with appropriate targeting to promote the identification of new testers and populations less likely to otherwise test, are necessary to ensure that the intervention reaches the target population.
\end{abstract}

treatment and viral load suppression targets are unlikely to be met. Over the past three decades, there has been considerable scale-up of HTS in sub-Saharan Africa. At the end of 2015, the Joint United Nations Programme on HIV and AIDS estimates indicated that approximately $23 \%$ of all people living with HIV (PLHIV) in East and Southern Africa were unaware of their status. However, at the 
end of 2018, this figure was 15\%. ${ }^{12}$ In Zambia, the 2016 Population-Based HIV Impact Assessment (ZAMPHIA) survey found annual incidence of HIV was around $0.67 \%$, with around 47000 new cases of HIV in adults expected each year cite. ${ }^{3}$ However, only $42.0 \%$ of women and $34.7 \%$ of men aged $15-59$ years had tested for HIV in the last 12 months. ${ }^{3}$ Insufficient reach of HTS persists in Zambia despite substantial changes to local and international HIV testing guidelines over the past 15 years, ${ }^{4-7}$ and is likely impeding uptake of HIV treatment and prevention services among those who need them.

To increase HIV testing coverage, alternative strategies for delivering HTS are needed, particularly in settings and among populations where the number of people with undiagnosed HIV and new infections is high. HIV self-testing (HIVST), whereby an individual tests themselves and interprets the result in private, is one option for scaling up HTS, and is recommended by the WHO as a tool for increasing HIV testing uptake. ${ }^{8}$ HIVST is particularly promising for reaching population subgroups with limited access to standard HTS, including men and adolescents. ${ }^{9-13}$ Various HIVST distribution strategies have been shown to increase uptake of HTS, including providing HIVST kits to pregnant women to reach their male partners, facility-based distribution, and door to door and distribution through social networks. ${ }^{12} 1415$ HIVST is also an acceptable way to increase HIV testing uptake and frequency among key populations, including female sex workers ${ }^{1617}$ and men who have sex with men. ${ }^{18}$

The UNITAID/PSI STAR (HIV Self-Testing AfRica) project was established to evaluate whether HIVST could increase HIV testing and support access to HIV care, treatment and prevention services in Africa, particularly among men and adolescents. ${ }^{19} 20$ This study reports the results of a pragmatic cluster randomised trial to investigate the impact of HIVST when distributed by community-based distributors (CBDs) in Zambia.

\section{METHODS}

\section{Trial design and participants}

We conducted a pair-matched cluster randomised trial of distribution of oral HIVST kits via CBDs. HIVST distribution was managed by the Society for Family Health (SFH) in Zambia. Clusters consisted of the catchment areas of health facilities in four districts in Zambia: Lusaka (two clinics, urban), Choma (four clinics, two urban and two rural), Kapiri Mposhi (four clinics, two urban and two rural) and Ndola (two clinics, urban). Selection of clusters was purposive and completed after consultation with the District Health Offices. Matching of the clusters was based on district and, within districts with four clusters, on distance from the clinic to the District Health, availability of antiretroviral therapy (ART) and HTS at the facility, and catchment population size. Total population in these clusters in 2010 ranged from 7933 to $58246 .^{21}$ Within clusters, all residents aged 16 years and older were eligible to receive HIVST kits. The protocol for this study has been published elsewhere. ${ }^{22}$ There were no important changes to the methods after the trial began.

\section{Data collection}

Household survey data

We used population-based cross-sectional survey data to assess the impact of the HIVST intervention. Intervention roll-out was staggered. The endline survey was completed at least 12 months after implementation commenced in each matched cluster pair, with the first clusters receiving the endline survey in October 2017 and the last in January 2018. A baseline survey was also conducted to assess balance on important cluster characteristics before the intervention began.

Sampling was conducted differently in rural and urban clusters. In rural clusters, we randomly selected standard enumeration areas defined by the Central Statistical Office and all households in these areas were approached to participate in the survey. In urban clusters, standard enumeration areas were subdivided into smaller blocks of 20-30 households. Blocks were randomly numbered and the first 12 were selected for data collection. Random sampling of blocks was done separately for baseline and endline surveys. Trained survey enumerators collected data using electronic data collection devices. A random $20 \%$ subsample of participants were asked an extended survey with additional questions on stigma, social harms and intimate partner violence (IPV).

At the household, survey enumerators sought permission from the head of household or representative to the head of the household to administer the household survey. Individual written informed consent was obtained from participants willing to complete an individual questionnaire. No return visits were conducted for eligible individual household members absent at the time of the household visit.

\section{ART data}

Data on the number of individuals initiating ART in each month before and during the intervention were collected from health facility databases by research staff. For all health facilities, we had at least 5 months of data prior to the start of intervention, and at least 11 months of data during the intervention period.

\section{Programme data}

Throughout the intervention period, CBDs and implementation supervisors from SFH recorded the number of HIVST kits distributed in each of the six intervention clusters by all CBDs. Data were available on the number of HIVST kits distributed by sex, age and to first-time testers.

\section{The HIVST intervention}

The HIVST intervention was delivered at cluster level. In the six clinic catchment areas randomly allocated to receive the HIVST intervention, HIVST kits were 
distributed by CBDs using a pragmatic distribution strategy, with CBDs distributing HIVST under typical programmatic conditions with limited researcher input. CBDs were trained on strategies for distribution. HIVST kits were also distributed by one distributor in each health facility. Within the community, CBDs went door to door distributing HIVST kits to residents. They also distributed HIVST kits to people found in high-density areas, such as markets or bus stops. In three of the six clusters, trained voluntary medical male circumcision (VMMC) promoters also distributed HIVST kits. There was no limit to the number of HIVST kits available in each cluster.

Eligible individuals ( $\geq 16$ years) were offered HIVST kits, and were able to receive one kit per CBD visit for their own use. Individuals were given a package consisting of an OraQuick HIV Self-Test labelled with a unique barcode, manufacturer's instructions for use translated into local language, a self-completed referral card for linkage to post-test services and an envelope for returning the kit after use. They were also given demonstrations of how to use kits, read and interpret results. Participants were encouraged to return used kits to boxes located in the community and at the local health facility. Demand generation for HIVST was conducted in HIVST intervention areas, and included monthly drama performances and announcements through public address systems and radios; and semimonthly distribution of fliers, brochures and posters. At the beginning of the intervention period, CBDs and VMMC mobilisers were paid a monthly stipend (US\$83 for CBDs and facility-based distributors and US $\$ 50$ for VMMC mobilisers). However, in March 2017 this was changed to a pay-for-performance structure. After this point, CBDs and VMMC mobilisers were incentivised based on the number of HIVST kits distributed and returned (US\$0.56/distributed kit and US\$0.28/ used kit returned).

HIVST implementation was staggered across clusters, with implementation beginning in Lusaka in September 2016, Choma in October 2016, Kapiri Mposhi in December 2016 and Ndola in January 2017. HIVST distribution continued for at least 1 year in each cluster. In clusters randomised to standard of care (SOC), clusters continued to receive HTS as provided by the health facility and other providers within each cluster.

\section{Primary and secondary outcomes}

The primary outcome was self-reported recent HIV testing, defined as testing for HIV in the 12 months prior to the date of the endline survey. The denominator included all individuals aged 16 years or older who consented to participate in the survey and had no missing data on whether they ever HIV tested. The numerator included individuals who reported one or more HIV tests in the 12 months prior to the survey.

Secondary outcomes included:

- HIV testing within 12 months after the intervention start date in each cluster. This outcome was included to aid the comparability of results of this cluster randomised trial with a similar trial in Malawi using this outcome. ${ }^{23}$

- Ever tested for HIV, which was chosen to highlight possible improvements in accessing previously untested populations using HIVST.

- Self-reported current ART use.

- Men circumcised in the last 12 months.

- ART initiation at clinic during the intervention period. Apart from ART initiation, which was collected using clinic data, all outcomes were based on individual self-report. Outcomes were measured at cluster level.

\section{Process indicators and population assessment of harms and benefits}

A community-based social harms reporting system was implemented in both HIVST and SOC areas. Household survey data also included process indicators on the distribution of HIVST and on possible harmful outcomes related to the HIVST intervention, including experience of IPV ${ }^{24}$ stigma, ${ }^{25}{ }^{26}$ self-reported forced HIV testing and forced disclosure of results.

\section{Sample size calculation}

The sample size was calculated using standard methods for cluster randomised trials ${ }^{27}$ and assumed $80 \%$ power to detect a $50 \%$ relative increase in the proportion of individuals self-reporting a recent HIV test to $75 \%$, with $95 \%$ confidence and cluster coefficient of variation $(\mathrm{k})$ of 0.2. Using 2013-2014 Demographic and Health Surveys data, baseline levels for individuals tested in the last 12 months were estimated to be $29 \%$ among men and $57 \%$ among women; we assumed a baseline recent HIV testing prevalence of $50 \%$ for the sample size calculation. For a two-sample comparison of matched proportions across six pairs of matched communities, we estimated that it would be necessary to recruit around 400 respondents per community, or 4800 respondents in total.

\section{Randomisation and blinding}

Within matched pairs, clusters were randomised 1:1 to receive either the HIVST intervention or SOC. Stata V.15.1 (College Station, Texas, USA) was used by BH to generate the random allocation sequence.

Cluster-level consent was obtained from the district medical office, management at each health facility and the neighbourhood health committees representing community members. Sensitisation activities were conducted in all communities before starting the study.

Due to the nature of the intervention, it was not possible to mask study participants or CBDs to intervention status. Survey and clinic data were collected by data collectors recruited and trained independently of SFH. Investigators were masked to allocation until after analysis of the primary outcome was complete.

\section{Statistical methods}

Analyses were conducted using standard methods for the analysis of a pair-matched cluster randomised trial with 
small number of clusters. ${ }^{27}$ Analyses were completed by intention-to-treat, using cluster-level summaries and according to a prespecified analysis plan.

Baseline data were collected to assess balance across key characteristics associated with the outcome, including baseline recent and ever HIV testing, self-reported HIV status, and sociodemographic factors including age and sex. We used baseline data to identify imbalances across arms by age, sex and assets index. As these covariates were unlikely to have been affected by the intervention, we adjusted the analysis using values for these covariates measured at endline. At baseline, clusters were balanced by recent and ever HIV testing.

For unadjusted analyses, risk ratios (RRs) for each arm were calculated as geometric mean of cluster-level RRs, and a paired t-test used to calculate $\mathrm{p}$ values. For adjusted analyses, a two-stage process was used to adjust for imbalances across arms. Prespecified subgroup analyses were conducted to determine whether the effect of the intervention on the primary outcome is modified by sex, age (younger than 25 years; 25 years and older) and educational attainment. ${ }^{28}$

A separate analysis was undertaken to estimate any impact on monthly ART uptake at clinics by arm, using clinic-level data on the number of ART initiations in each month before and during the implementation period. For this analysis, the denominator was the overall population of the clinic catchment area estimated using census data. ${ }^{21}$ Ordinary least squares regression was used to estimate the effect of the intervention on logged number of ART initiations per month per 1000 population, and the regression model adjusted for clinic pair and total logged ART initiations per 1000 in the $5-10$ months prior to the intervention start date.

All analyses were conducted using Stata V.15.1 and adhere to Consolidated Standards of Reporting Trials reporting guidelines for the reporting of cluster randomised trials. ${ }^{29}$

\section{Process evaluation}

In addition to the analysis of impact, we conducted a retrospective quantitative process evaluation using survey and programme data to understand delivery and reach of the HIVST intervention. ${ }^{30}$ To guide the process evaluation, we developed a simple framework of how the intervention was expected to have an impact on the recent HIV testing (online supplemental figure 1).

\section{Patient and public involvement}

Community sensitisation meetings were conducted before the beginning of data collection, and formative research was conducted among key stakeholders to assess the acceptability of HIVST distribution in the community. A social harms reporting system including CBDs, local health clinics and traditional leaders was established as part of the trial. Community members were not involved in the research design or analysis of data.

\section{RESULTS}

\section{The effect of the HIVST intervention on recent HIV testing}

Twelve pair-matched clusters were randomised (figure 1). The total population in the study area was 308 822, with a median population of 15465 per cluster (range: $7673-58$ 246). At baseline, self-reported HIV prevalence was $8.4 \%$ $(42 / 500)$ in the HIVST intervention arm and $8.3 \%$ $(44 / 528)$ in the SOC arm, and recent testing coverage was $63.3 \%(1457 / 2272)$ in the HIVST arm and $64.1 \%$ $(1496 / 2364)$ in SOC arm (table 1). The endline survey included 5005 participants. Participation in the endline survey was similar across arms, with $60.1 \%$ (2528/4202) of eligible household members participating in intervention arm and $61.5 \%(2477 / 4027)$ in SOC arm. Participant characteristics were balanced across arms, though slightly more respondents in the SOC arm were in the lowest assets quintile. Across both arms, survey participation was higher among women than men. In both arms, $2.2 \%$ of survey respondents did not provide information on HIV testing history and were omitted from the primary analysis (56/2528 in intervention and 55/2477 in SOC).

At endline, $65.8 \%(1626 / 2472)$ of respondents in the HIVST arm had recently tested compared with $60.0 \%$ $(1452 / 2422)$ in the SOC arm, with no evidence of a difference between arms (adjusted RR: 1.08, 95\% CI: 0.94 to $1.25 ; \mathrm{p}=0.224$; table 2 ). There was no evidence of a difference between arms in any secondary outcomes (table 2). Subgroup analyses showed no statistical evidence for differences in recent testing by arm by respondent sex, age or education (online supplemental table 1).

\section{Delivery of the HIVST intervention}

The intervention was delivered in facilities and the community in all six intervention clusters. SFH trained 60 CBDs to distribute HIVST kits across these six clusters, with 12 CBDs per cluster in urban areas and 8-10 per cluster in rural areas (table 3). CBDs distributed 65585 HIVST kits during the intervention; of these, $53626(82 \%)$ were distributed in the community and the remainder in health facilities $(8378 ; 13 \%)$ or by VMMC mobilisers $(3581 ; 5 \%)$. The number of HIVST distributed per capita varied across clusters. The percentage of HIVST kits distributed to men ranged from $45.2 \%$ $(3985 / 8823)$ to $56.0 \%$ (1384/20 322), and distribution to first-time testers ranged from $10.7 \%(696 / 6487)$ to $29.1 \%(803 / 1768)$.

\section{Reach of the HIVST intervention}

Relative to the SOC arm, more individuals in the intervention arm reported that they had seen a CBD in their community, had a CBD come to their household, and had ever or recently self-tested for HIV (table 4). However, the proportion reporting being aware of the HIVST intervention arm was lower than expected after 12 months of ongoing distribution. Only $58.0 \%$ (1462/2520) of respondents in the HIVST intervention arm were aware of HIVST, compared with 28.3\% (700/2474) in SOC arm. Of households in the HIVST arm who reported being 


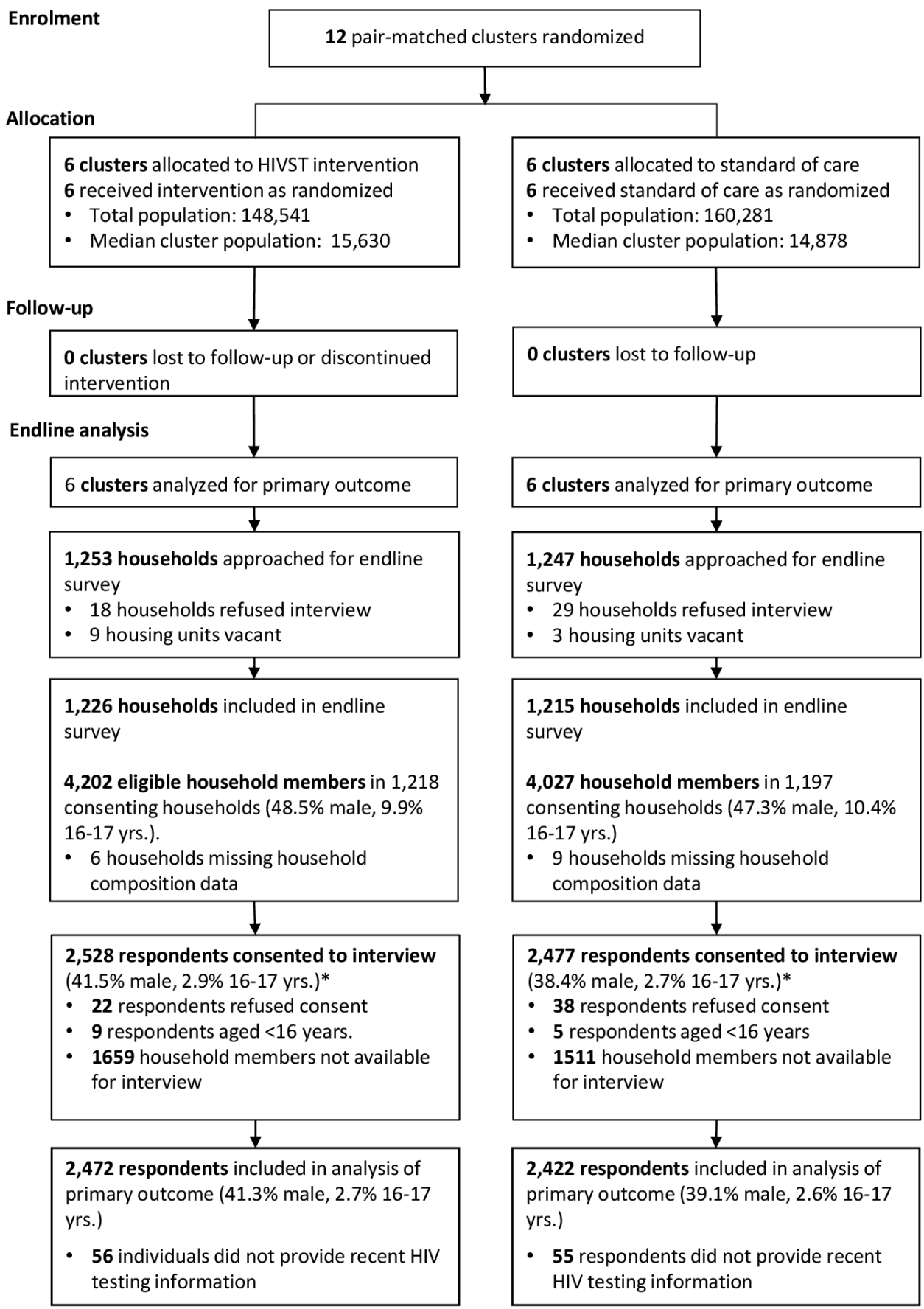

* 12 respondents in the intervention arm and 17 from standard of care arm did not have household composition data and are excluded from the household member count.

Figure 1 CONSORT diagram showing flow of study participants. CONSORT, Consolidated Standards of Reporting Trials; HIVST, HIV self-testing.

visited by CBDs, $75 \%$ (456/608) of respondents reported receiving an HIVST kit, and $75 \%$ of these $(369 / 456)$ used the HIVST. Only $23.5 \%$ (585/2493) of respondents reporting HIVST during the intervention period.

\section{Social harms and stigma}

No social harms were identified during the study implementation period. There were no differences in selfreported IPV or in HIV stigma by arm. In adjusted analyses, respondents in the HIVST were more likely to report there was pressure to test for their most recent HIV test regardless of testing mode $(2.2 \%(43 / 1946)$ in intervention and $1.3 \%(23 / 1802)$ in SOC).

\section{DISCUSSION}

We found no evidence that community-based distribution of HIVST by CBDs in community settings and at facilities increased population levels of recent HIV testing or had an impact on secondary outcomes. Although HIVST 
Table 1 Characteristics of household survey respondents

HIVST intervention, $\mathbf{N}(\%) \quad$ SOC, $\mathrm{N}(\%)$

\begin{tabular}{|c|c|c|}
\hline \multicolumn{3}{|l|}{ Baseline } \\
\hline Total & 2433 & 2446 \\
\hline Self-reported HIV positive status $(n=1056)$ & $42(8.4)$ & $44(8.3)$ \\
\hline Ever tested for HIV† & $1971(82.2)$ & $1918(83.2)$ \\
\hline Tested for HIV within past 12 months $\ddagger$ & $1496(63.3)$ & $1457(64.1)$ \\
\hline \multicolumn{3}{|l|}{ Endline } \\
\hline Total households & 1224 & 1206 \\
\hline \multicolumn{3}{|l|}{ Household characteristics } \\
\hline \multicolumn{3}{|l|}{ Assets index (no (\%))§ } \\
\hline Lowest & 345 (31.0) & 397 (36.9) \\
\hline Second & $386(34.7)$ & 367 (30.9) \\
\hline Highest & 392 (34.2) & 359 (32.2) \\
\hline Individual characteristics & 2528 & 2477 \\
\hline Age (mean (SD)) & $32.7(14.1)$ & $33.8(14.8)$ \\
\hline \multicolumn{3}{|l|}{ Age group (no (\%)) } \\
\hline $16-19$ years & $354(14.0)$ & $363(14.6)$ \\
\hline 20-24 years & $567(22.4)$ & $475(19.2)$ \\
\hline $25-29$ years & $439(17.4)$ & $404(16.3)$ \\
\hline 30-39 years & $507(20.1)$ & $527(21.3)$ \\
\hline 40-49 years & $315(12.5)$ & $321(13.0)$ \\
\hline $50-59$ years & $193(7.6)$ & $217(8.8)$ \\
\hline $60+$ years & $153(6.0)$ & $170(6.9)$ \\
\hline Male (no/\%) & 1048 (41.5) & 970 (39.2) \\
\hline \multicolumn{3}{|l|}{ Marital status (no/\%)ף } \\
\hline Married or living as married & $1376(54.5)$ & $1383(56.0)$ \\
\hline Never married & $866(34.3)$ & $806(32.6)$ \\
\hline Widowed/separated/divorced & $282(11.2)$ & $281(11.4)$ \\
\hline \multicolumn{3}{|l|}{ Education $(\mathrm{no} / \%)^{\star \star}$} \\
\hline No or primary education & $1275(50.5)$ & $1207(48.8)$ \\
\hline Secondary incomplete & $836(33.1)$ & 832 (33.6) \\
\hline Completed secondary or higher & $416(16.5)$ & 437 (17.6) \\
\hline
\end{tabular}

*HIV status asked of $20 \%$ subsample only ( $n=511$ in intervention areas and $n=545$ in comparison areas); 28 respondents declined to respond.

†Ever tested information missing for 176 respondents.

$\ddagger$ Tested in past 12 months; information missing for 243 respondents.

$\S$ Assets missing for 244 households and 442 respondents.

१Marital status missing for 11 respondents.

${ }^{\star \star}$ Education missing for two respondents.

HIVST, HIV self-testing; SOC, standard of care.

awareness was higher in the intervention arm, HIVST awareness remained low, and few individuals reported ever self-testing. Among the respondents visited at home by CBDs, most had accepted and used HIVST.

Other trials of community-based HIVST delivery have shown an impact on increasing population testing coverage. A companion STAR study of a similar HIVST intervention in rural Malawi found a 33\% relative increase in population levels of recent testing after
1 year of HIVST distribution, with a $76 \%$ increase among persons $>20$ years old and a $50 \%$ increase among men. ${ }^{23}$ In Zambia, a cluster randomised trial of the door-to-door offer of HIVST as an option for HIV testing, nested in the HPTN 071 (PopART) trial of universal testing and treatment, found a small but significant impact of the HIVST intervention on knowledge of HIV status. ${ }^{12}$ The effect of this nested intervention was driven by an impact among men, with no evidence of an effect among women. The 


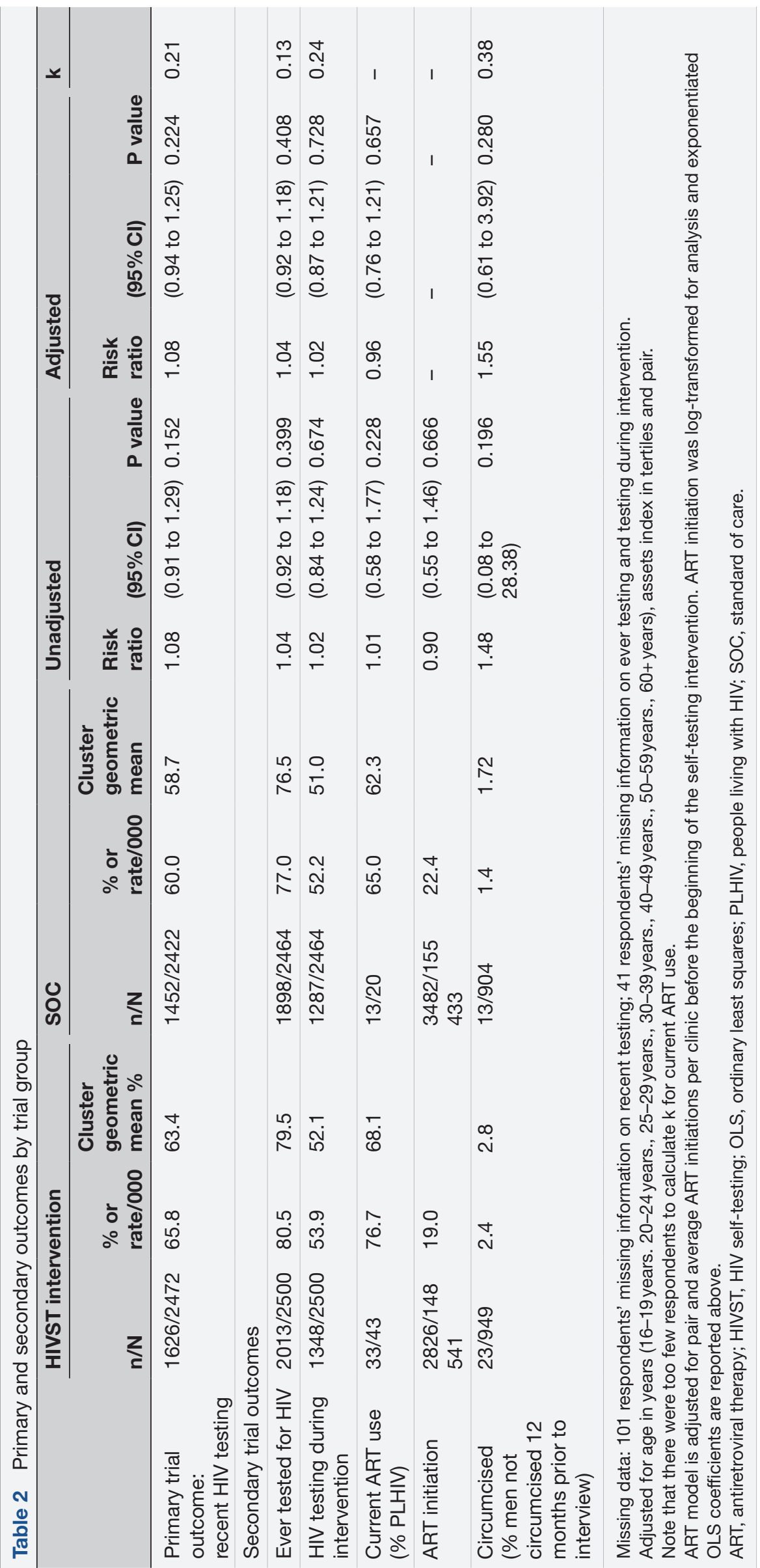

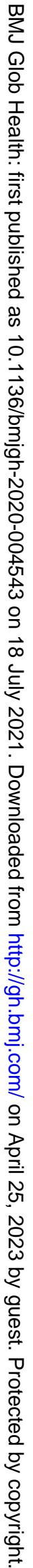




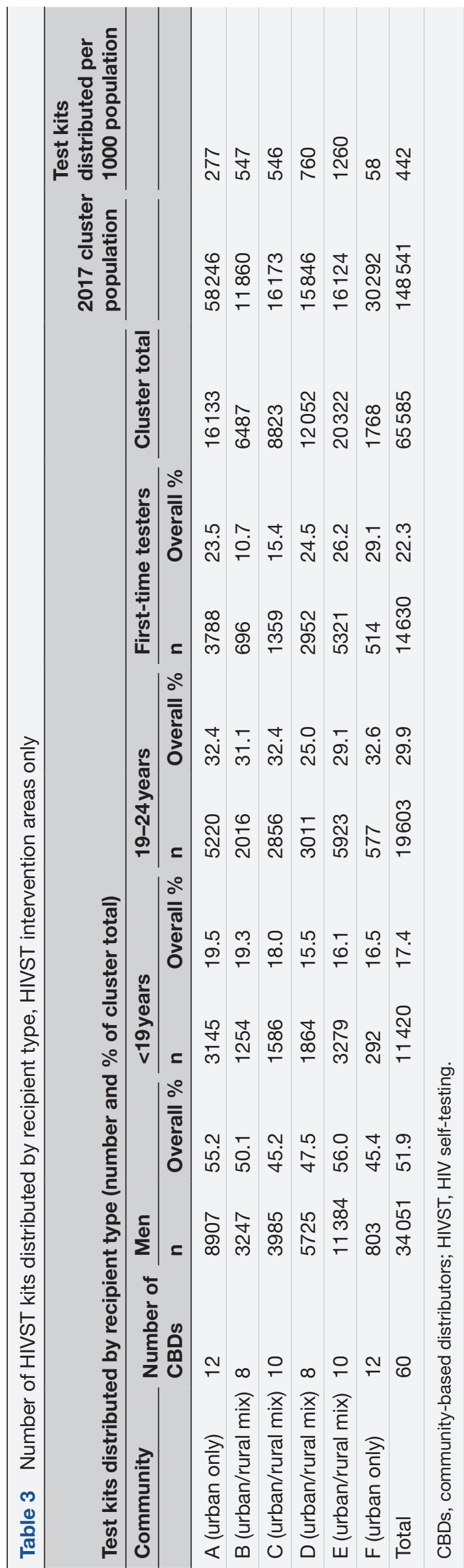

offer of an HIVST to women for distribution to a partner absent at the time of the household visit contributed to this effect among men. In another STAR-supported study in rural Zimbabwe, a non-randomised comparison of areas with and without HIVST distribution campaigns found a $27 \%$ increase in ART initiations at local clinics during the campaign, suggesting that HIVST users in Zimbabwe were able to link successfully following a reactive HIVST. $^{31}$

This discrepancy in findings between our study and other evaluations of community HIVST distribution may be due to the low dose of intervention provided at population level, as levels of implementation are known to affect observed outcomes. ${ }^{32}{ }^{33}$ In intervention clusters, only $58 \%$ had heard of HIVST, and among these only $62 \%$ reported a CBD visiting their home. In the companion trial in Malawi, $88 \%$ of individuals sampled in the intervention arms had heard of HIVST compared with $29 \%$ in the control arms. ${ }^{23}$ Exposure to HIVST among area residents was thus lower than anticipated in intervention areas, leading to low HIVST uptake. Further, in some clusters few HIVST kits were distributed per capita, particularly in urban clusters. Taken together, these process measures suggest that HIVST may not have been accessible to all residents in intervention areas, hampering the ability to identify changes in the outcome.

Alternatively, an intervention may not show an impact because theories underlying the causal pathway linking the intervention with the expected outcome may be incorrect. ${ }^{30}$ Community-based distribution was expected to increase knowledge and acceptability of HIVST, remove barriers to accessing HTS and thereby increase coverage of HTS. In this study, CBDs distributed HIVST kits both door to door and in high-density areas. This distribution method may not have been as acceptable to community members as expected, so they may have been unlikely to learn about or receive HIVST from a CBD. Finally, as a novel technology at the time of the trial, more time may have been required for more individuals to consider HIVST as an acceptable way to test for HIV. As data to explore these specific domains were not collected at the time of the trial, we can only speculate whether acceptability affected the observed findings.

This intervention showed no impact on testing coverage among priority populations for HIVST: young people and men. HIVST has been shown to be highly acceptable among young people and men, including in Zambia. ${ }^{9} 101234$ There is no evidence that this was different in this study, as nearly all survey respondents who received an HIVST kit reported using the test. However, in this intervention, HIVST may have been less accessible to key target subgroups than anticipated. Other models of HIVST distribution may be more effective at reaching these key subgroups. Secondary distribution models targeting the partners of antenatal care clients have been effective in increasing testing coverage, ${ }^{14}$ and secondary distribution strategies capitalising on social networks have been used successfully 


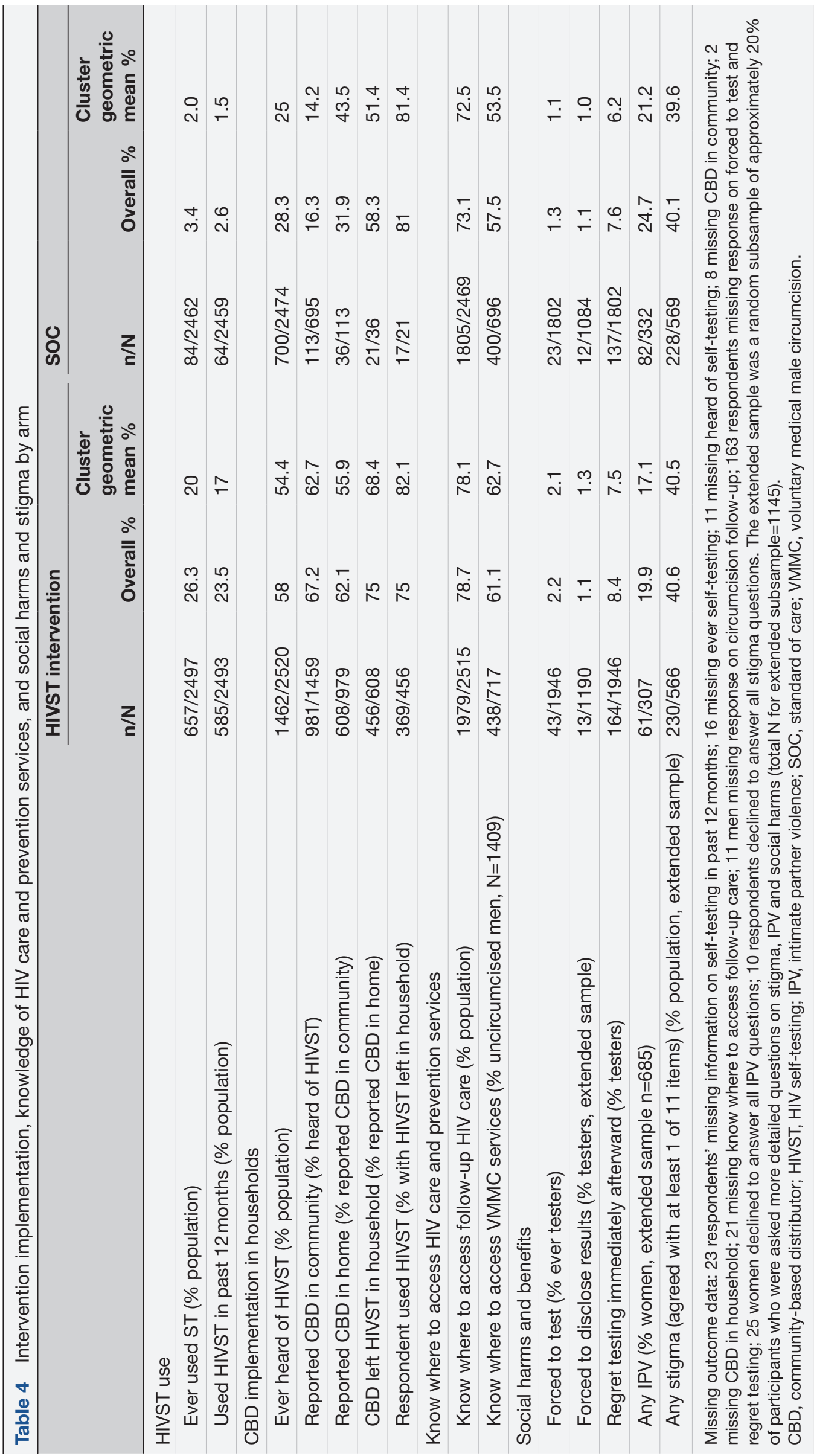


among adolescents and other key populations. ${ }^{35-37}$ Workplace distribution may also target men effectively, while distribution in family planning clinics, universities, clubs and other youth-friendly venues may make HIVST more accessible to adolescents. ${ }^{38}{ }^{39}$ Community-led distribution strategies that include community members in the design and implementation of HIVST distribution programmes may also increase the acceptability and uptake of HIVST, particularly among otherwise underserved populations. ${ }^{40}$

This study has several limitations. Prevalence of recent testing for HIV at baseline was high in the study communities compared with estimates from the 2016 ZAMPHIA survey which the sample size was based on, leaving the study underpowered to detect differences in recent testing coverage. HIV testing and VMMC outcomes were measured using self-reported data, and are subject to misreporting, but were collected in the same manner in both arms and so are unlikely to have substantial bias. ART initiation was measured using clinic data and self-report. Although we were able to triangulate this outcome across data sources, we may have underestimated ART uptake as clinic catchment areas were larger than HIVST clusters, diluting clinic-level effects, and not all newly diagnosed PLHIV will have chosen their local clinic for HIV services leading to undercapture.

While HIVST has potential to increase testing uptake among populations unreached by currently available HTS, broad community-based distribution of HIVST was not successful in increasing testing coverage at population level in Zambia. HIVST distribution in high-density areas, door to door and in health facilities increased awareness of HIVST, but levels of ever HIVST remained low in intervention arm. Rigorous monitoring and evaluation of the distribution in real time, with distribution incentives promoting the identification of new testers and populations less likely to otherwise test, may have improved the reach and dose of the intervention and increased its effectiveness.

\section{Author affiliations}

${ }^{1}$ Department of Infectious Disease Epidemiology, MRC International Statistics and Epidemiology Group, London School of Hygiene and Tropical Medicine, London, UK ${ }^{2}$ Department of Clinical Research, London School of Hygiene and Tropical Medicine, London, UK

${ }^{3}$ Zambart, Lusaka, Zambia

${ }^{4}$ Society for Family Health, Lusaka, Zambia

${ }^{5}$ Population Services International, Cape Town, South Africa

${ }^{6}$ School of Public Health, University of the Witwatersrand, Johannesburg, South Africa

${ }^{7}$ Global HIV, Hepatitis and STI Programme, World Health Organization, Geneva, Switzerland

${ }^{8}$ Malawi-Liverpool-Wellcome Trust Clinical Research Programme, Blantyre, Malawi

Twitter Cheryl Johnson @ccasejohn

Contributors HA, ELC and KH conceptualised the study. HA, BH, KLF, ELC, NC, $\mathrm{NH}, \mathrm{CJ}, \mathrm{AM}, \mathrm{MNa}, \mathrm{MNe}, \mathrm{MS}$ and DT coauthored the study protocol. NC, MNa and GS led intervention implementation. NH and DT led data collection. BH, MNe and KLF conducted statistical analysis. MNe wrote the first draft of the manuscript. All authors contributed to writing and editing the manuscript, and have reviewed and approved this version.
Funding This work is supported by Unitaid (grant number: P0\#8477-0-600). ELC is supported by the Wellcome Trust (grant number: WT091769).

Competing interests The authors have no competing interests. The contents in this article arethose of the authors and do not necessarily reflect the view of the WorldHealth Organization.

\section{Patient consent for publication Not required.}

Ethics approval The study was approved by the University of Zambia Biomedical Research Ethics Committee (UNZABREC; Ref.: 013-11-15) and London School of Hygiene and Tropical Medicine (LSHTM) ethics committees (ID: 10660). Permission to conduct the study was also granted by the Zambian National Health Research Authority and the Ministry of Health, Zambia.

Provenance and peer review Not commissioned; externally peer reviewed.

Data availability statement Data are available in a public, open access repository. Data are available upon request. Data available upon request from datacompass.Ishtm.ac.uk. The protocol is available from hivstar.Ishtm.ac.uk.

Supplemental material This content has been supplied by the author(s). It has not been vetted by BMJ Publishing Group Limited (BMJ) and may not have been peer-reviewed. Any opinions or recommendations discussed are solely those of the author(s) and are not endorsed by BMJ. BMJ disclaims all liability and responsibility arising from any reliance placed on the content. Where the content includes any translated material, BMJ does not warrant the accuracy and reliability of the translations (including but not limited to local regulations, clinical guidelines, terminology, drug names and drug dosages), and is not responsible for any error and/or omissions arising from translation and adaptation or otherwise.

Open access This is an open access article distributed under the terms of the Creative Commons Attribution IGO License (CC BY NC 3.0 IGO), which permits use, distribution, and reproduction in any medium, provided the original work is properly cited. In any reproduction of this article there should not be any suggestion that WHO or this article endorse any specific organization or products. The use of the WHO logo is not permitted. This notice should be preserved along with the article's original URL.

Disclaimer: The author is a staff member of the World Health Organization. The author alone is responsible for the views expressed in this publication and they do not necessarily represent the views, decisions or policies of the World Health Organization.

\section{ORCID iD}

Melissa Neuman http://orcid.org/0000-0002-8870-6504

\section{REFERENCES}

1 UNAIDS. Accelerating towards 90-90-90. Geneva: UNAIDS, 2018.

2 UNAIDS. Global AIDS update 2019 - communities at the centre. Geneva: UNAIDS, 2019.

3 ICAP. Zambia population-based HIV impact assessment. Karachi: ICAP, 2016.

4 World Health Organization. Consolidated guidelines on HIV testing services. Geneva: World Health Organization, 2015.

5 World Health Organization, UNAIDS. Guidance on provider-initiated HIV testing and counselling in health facilities. Geneva: World Health Organization, 2007.

6 Republic of Zambia Ministry of Health. Zambia consolidated guidelines for treatment and prevention of HIV infection. Zambia: Republic of Zambia Ministry of Health, 2018.

7 Republic of Zambia Ministry of Health. Zambia national guidelines for HIV counselling and testing. Zambia: Republic of Zambia Ministry of Health, 2006.

8 World Health Organization. Guidelines on HIV self-testing and partner notification. Geneva: World Health Organization, 2016.

9 Kalibala S, Tun W, Muraah TW. Knowing myself first: feasibility of self-testing among health workers in Kenya. Nairobi: Population Council, 2011.

10 Krause J, Subklew-Sehume F, Kenyon C, et al. Acceptability of HIV self-testing: a systematic literature review. BMC Public Health 2013;13:735.

11 Kurth AE, Cleland CM, Chhun N, et al. Accuracy and acceptability of oral fluid HIV self-testing in a general adult population in Kenya. AIDS Behav 2016;20:870-9.

12 Mulubwa C, Hensen B, Phiri MM, et al. Community based distribution of oral HIV self-testing kits in Zambia: a clusterrandomised trial nested in four HPTN 071 (PopART) intervention communities. Lancet HIV 2019;6:e81-92. 
13 Jamil MS. Effectiveness of HIVST distribution models in the general population in SSA: a systematic review. Kigali, Rwanda: ICASA, 2019.

14 Choko AT, Kumwenda MK, Johnson CC, et al. Acceptability of woman-delivered HIV self-testing to the male partner, and additiona interventions: a qualitative study of antenatal care participants in Malawi. J Int AIDS Soc 2017;20:21610.

15 Thirumurthy $\mathrm{H}$, Masters SH, Mavedzenge SN, et al. Promoting male partner HIV testing and safer sexual decision making through secondary distribution of self-tests by HIV-negative female sex workers and women receiving antenatal and post-partum care in Kenya: a cohort study. Lancet HIV 2016;3:e266-74.

16 Maman S, Murray KR, Napierala Mavedzenge S, et al. A qualitative study of secondary distribution of HIV Self-Test kits by female sex workers in Kenya. PLoS One 2017;12:e0174629.

17 Chanda MM, Ortblad KF, Mwale M, et al. Hiv self-testing among female sex workers in Zambia: a cluster randomized controlled trial. PLoS Med 2017;14:e1002442.

18 Figueroa C, Johnson C, Verster A, et al. Attitudes and acceptability on HIV self-testing among key populations: a literature review. AIDS Behav 2015;19:1949-65.

19 Hatzold K, Gudukeya S, Mutseta MN, et al. HIV self-testing: breaking the barriers to uptake of testing among men and adolescents in sub-Saharan Africa, experiences from STAR demonstration projects in Malawi, Zambia and Zimbabwe. J Int AIDS Soc 2019;22 Suppl 1:e25244.

20 Ingold $\mathrm{H}$, Mwerinde $\mathrm{O}$, Ross AL, et al. The Self-Testing Africa (StAR) initiative: accelerating global access and scale-up of HIV self-testing. $J$ Int AIDS Soc 2019;22 Suppl 1:e25249.

21 [Zambia] CSOC. 2010 census of population and housing. volume 11: national descriptive tables. Lusaka Central Statistical Office; 2010. http://catalog.insn.org/index.php/catalog/4124/download/55313 [Accessed 10 March 2021].

22 Neuman M, Indravudh P, Chilongosi R, et al. The effectiveness and cost-effectiveness of community-based lay distribution of HIV self-tests in increasing uptake of HIV testing among adults in rural Malawi and rural and peri-urban Zambia: protocol for StAR (self-testing for Africa) cluster randomized evaluations. BMC Public Health 2018;18:1234.

23 Indravudh P, Fielding K, Neuman M. Increasing knowledge of HIV status and demand for art using community-based HIV self-testing: a cluster randomised trial in rural Malawi. AIDS 2018.

24 Garcia-Moreno C, Jansen HAFM, Ellsberg M, et al. Prevalence of intimate partner violence: findings from the WHO multi-country study on women's health and domestic violence. The Lancet 2006;368:1260-9.

25 Hargreaves JR, Krishnaratne S, Mathema $\mathrm{H}$, et al. Individual and community-level risk factors for HIV stigma in 21 Zambian and South African communities: analysis of data from the HPTN071 (PopART) study. AIDS 2018;32:783-93.

26 Krishnaratne S, Hensen B, Cordes J, et al. Interventions to strengthen the HIV prevention cascade: a systematic review of reviews. Lancet HIV 2016;3:e307-17.
27 Hayes R, Moulton L. Cluster randomised trials. Boca Raton, FL, USA: CRC Press, 2009.

28 Cheung YB, Jeffries D, Thomson A, et al. A simple approach to test for interaction between intervention and an individual-leve variable in community randomized trials. Trop Med Int Health 2008;13:247-55.

29 Campbell MK, Piaggio G, Elbourne DR, et al. Consort 2010 statement: extension to cluster randomised trials. BMJ 2012;345:e5661.

30 Moore GF, Audrey S, Barker M, et al. Process evaluation of complex interventions: medical Research Council guidance. BMJ 2015;350:h1258.

31 Sibanda E, Neuman M, Tumushime M. Linkage to care after HIV self-testing in Zimbabwe: a cluster-randomized trial. Conference on retroviruses and opportunistic infections.. Conference on Retroviruses and Opportunistic Infections (CROI); March 2018, Boston, MA, USA, 2018.

32 Hargreaves JRM, Goodman C, Davey C, et al. Measuring implementation strength: lessons from the evaluation of public health strategies in low- and middle-income settings. Health Policy Plan 2016;31:860-7.

33 Durlak JA, DuPre EP. Implementation matters: a review of research on the influence of implementation on program outcomes and the factors affecting implementation. Am J Community Psychol 2008;41:327-50.

34 Choko AT, MacPherson P, Webb EL, et al. Uptake, accuracy, safety, and linkage into care over two years of promoting annual SelfTesting for HIV in Blantyre, Malawi: a community-based prospective study. PLoS Med 2015;12:e1001873.

35 Lippman SA, Lane T, Rabede O, et al. High acceptability and increased HIV-testing frequency after introduction of HIV Self-Testing and network distribution among South African MSM. J Acquir Immune Defic Syndr 2018;77:279-87.

36 Conserve DF, Alemu D, Yamanis T, et al. "He told me to check my health": a qualitative exploration of social network influence on men's HIV testing behavior and HIV self-testing willingness in Tanzania. Am J Mens Health 2018;12:1185-96.

37 Shahmanesh M, Mthiyane T, Herbst C. Effect of Peer-distributed HIV Self-Test kits on demand for biomedical HIV prevention in rural KwaZulu-Natal, South Africa: a three-armed cluster-randomised trial comparing social-networks versus direct delivery. HIVR4P. virtual. HIVR4P; January 2021, virtual, 2021.

38 van Rooyen $\mathrm{H}$, Tulloch $\mathrm{O}$, Mukoma $\mathrm{W}$, et al. What are the constraints and opportunities for HIVST scale-up in Africa? Evidence from Kenya, Malawi and South Africa. J Int AIDS Soc 2015;18:19445.

39 Indravudh PP, Sibanda EL, d'Elbée M, d'Elbée M, et al. 'I will choose when to test, where I want to test': investigating young people's preferences for HIV self-testing in Malawi and Zimbabwe. AIDS 2017;31 Suppl 3:S203.

40 Indravudh PP, Fielding K, Kumwenda MK, et al. Community-led delivery of HIV self-testing targeting adolescents and men in rura Malawi: a cluster-randomised trial. Journal Of The International Aids Society 2019;22:94-5. 